using Quotient testing. Researchers at the King's College London Institute of Psychiatry, led by molecular psychiatrist Philip Asherson, are now running a trial in a UK prison population with ADHD; their goal is to determine whether early changes seen in the QbTest after the first one or two doses of medication correlate with long-term clinical outcomes more strongly than clinical interviews do. Meanwhile, psychologist Hanna Christiansen and her colleagues at the Philipp University of Marburg in Germany are now assessing the usefulness of the QbTest in people newly diagnosed with ADHD over the age of 50 .

Both QbTest and Quotient still rely on some aspect of behavior, though, and many researchers would like to see ADHD diagnoses made with a more rigorous biomarker, either on a brain scan or by a blood test. That could provide the ultimate objective assessment of neurobiological pathology.

A vast amount of research is ongoing into biomarkers for ADHD. In the meantime, Teicher notes that the available computerized attention tasks might be reflecting deeperseated biology in the brain. In a 2000 report in Nature Medicine (Nat. Med. 6, 470-473, 2000), he and his colleagues showed that blood volume in the striatum, as measured by magnetic resonance imaging, strongly correlated with young boys' scores on motion analysis tests. "It gives you a window into brain function," says Teicher, who maintains a financial stake in Quotient (which is marketed by the global learning company Pearson).

As for the young patient at the Focus-MD clinic, QbTest indicated that the boy was "absolutely rock-solid normal" in terms of attentiveness and hyperactivity, Wiley says. $\mathrm{He}$ didn't need stimulant medications. $\mathrm{He}$ didn't have ADHD. What he did have was dyslexia, a disorder that can resemble ADHD when clinicians rely only on verbal and written clinical measurements. Thus, for Wiley, QbTest "gave me the confidence to tell this mother, 'I think we're barking up the wrong tree."

Elie Dolgin

\title{
Mystery around drug adherence still plagues medical literature
}

The randomized controlled trial, or RCT, represents the gold standard of interventional studies of new drugs. But how reliable are the results when it remains unknown whether subjects in the trial actually took their medicines at all? A 2007 analysis found that only $33 \%$ of 192 papers describing RCTs of oral therapies for six chronic diseases disclosed adherence results (Am. J. Med. Sci. 334, 248-254, 2007). Now, in light of new data suggesting a continued lack of information about drug adherence in the medical literature, some researchers are calling for a reform of reporting guidelines.

"Failure to find significant results when they actually exist between treatment arms, underestimation of a drug's efficacy or side effects and overestimation of the effective dose are all potential undesirable consequences of inadequate adherence in drug trials," says William Robiner, a clinical health psychologist who studies drug adherence at the University of Minnesota Medical School in Minneapolis. "The truth is we don't know enough about patient adherence in RCTs-adherence is a weak link in clinical research."

In a study that appears in the April issue of the journal Clinical Trials (11, 195-204, 2014), Walter Kernan and his colleagues at the Yale School of Medicine in New Haven, Connecticut, report that only $46 \%$ of 111 oral therapy RCTs published in ten high-impact medical journals in 2010 reported patient drug adherence. Notably, studies that reported adherence were more likely to also report negative findings.

Given the low rates of adherence reporting, some researchers see the US Food and Drug Administration (FDA) as the solution. Currently, the FDA provides soft guidelines on clinical trial conduct, but it only encourages, not requires, accurate adherence monitoring. "The FDA should have explicit requirements on this," says Terrence Blaschke, emeritus professor of medicine at Stanford University who has studied drug adherence and served as a chair and member of drug advisory committees for the FDA.

\section{In the papers}

Others say that the onus should fall on the medical journals that report RCTs. Yet Jeffrey Drazen, editor-in-chief of the New England Journal of Medicine, is more equivocal about whether publications should include adherence data. "All the randomized clinical trials [we] published for the past two years have their protocols on [our] website," says Drazen, who adds he does not have a strong opinion on whether patient drug adherence should be reported in medical journal's papers reporting clinical trial results.

Kernan believes that drug adherence reporting in publications will not improve until it is required as part of the standards set by the CONSORT (Consolidated Standards of Reporting Trials) group, which has been publishing and updating a guideline on trial data disclosure since 1993. "This guideline is policy setting," says Kernan, "For publication in top medical journals, [investigators] adhere to these guidelines." At present, CONSORT does not provide a firm stance on whether investigators should report patient adherence. (Nature Medicine and other Nature journals ask that authors follow the CONSORT guidelines.)

According to Kenneth Schulz, lead author of the most recent CONSORT guidelines, excluding nonadherent patients can actually lead to biased results-ones that do not reflect how patients take their oral medications in the real world. This is also the FDA's rationale for using intent-to-treat analysis (ITT) to evaluate most RCTs. ITT incorporates all randomized patients regardless of whether they adhered to the study protocol, and the analysis is thought to mimic real-world effectiveness. This helps avoid reliance on self-reported adherence information from patients, which is usually "terrible," says Schulz, and never used. "Too many trial resources are devoted to monitoring adherence," he adds. However, his detractors point out that although ITT is the standard, if too many patients in the treatment arm are nonadherent, this can bias the results towards a negative outcome.

Ultimately, Kernan holds out hope that the scientific community-including researchers, funding and regulatory agencies and medical journal editors - will one day reach a consensus on both terminologies and standards for detailing drug adherence in RCTs. "Improved reporting of adherence will enhance the value of clinical trial research," he says, "and everyone would benefit from this development."

Anna Azvolinsky 